\title{
Reconstruction of transient pressure and acceleration over a tire surface using the inverse time domain boundary element method
}

\author{
Yang Zhang, Chuanxing Bi, Xiaozheng Zhang*, Yongbin Zhang, and Liang Xu \\ Institute of Sound and Vibration Research, Hefei University of Technology, 193 Tunxi Road, Hefei 230009, People's Republic of China
}

\begin{abstract}
The inverse time domain boundary element method (ITBEM) that is derived from the direct time domain boundary element method by eliminating the retarded time is able to reconstruct the transient pressure and flux on the surface of an arbitrarily shaped source by measuring the pressure on a hologram surface. In the present work, the ITBEM is applied to reconstruct the transient pressure and acceleration over the surface of a tire which is supported away from the ground in a semi-anechoic chamber. The tire is impacted by a rigid sphere to generate a transient sound field, and the measurement is controlled by a trigger which is connected to an acceleration sensor stuck on the surface of the tire. The pressure and acceleration on the surface of the tire are reconstructed from the holographic pressure measured by array microphones. By visualizing the pressure and acceleration with respect to the elapsed time, the wave propagation phenomenon of the pressure and acceleration on the surface of the tire is shown clearly. The comparison of the reconstructed surface acceleration to the measured one demonstrates the effectiveness of ITBEM for transient sound field reconstruction.
\end{abstract}

\section{Introduction}

Nearfield acoustic holography (NAH) was first documented in the early 1980s [1,2] for the reconstruction of acoustic field based on the measurement of acoustic pressures on a hologram plane. The conventional NAH was carried out via Fourier acoustics and it achieved great success in analysing sources of planar, cylindrical, and spherical shapes [3]. In order to reconstruct the acoustic field radiated by arbitrarily shaped sources, the inverse boundary element method (IBEM) was proposed in the late 1980s $[4,5]$. Since then, a large number of studies on the IBEM-based NAH have emerged.

Use Boundary element method can not only analyse stationary problems in frequency domain but also can simulate transient problems in time domain, which is usually called time domain boundary element method (TBEM). Many researches on acoustic radiation and scattering problems have been conducted using the TBEM [6-10], and the widely known instability problem of TBEM has also been deeply studied [10-13]. Recently, an inverse TBEM (ITBEM) was developed for transient sound field reconstruction by Zhang et al. [14]. They combined the surface and field time domain integral equations and shifted the time axis of the field integral equation to rule out the retarded time, and then solved the combined equations implicitly in a marching-on-intime (MOT) way. In the ITBEM, the truncated singular value decomposition (TSVD) method was applied to suppress the measurement noise in order to acquire stable results.
In the present paper, the ITBEM is applied to reconstruct the transient pressure and acceleration over an impacted tire surface from the measured pressure. The reconstruction results are visualized with respect to the elapsed time. The effectiveness of ITBEM is then illustrated through the comparison of the measured and reconstructed accelerations.

\section{Theory of ITBEM}

The ITBEM is based on the time domain boundary integral equation which can be expressed as [15]

$$
\begin{aligned}
& c(\mathbf{x}) \varphi(\mathbf{x}, t)+\int_{\Gamma} \int_{0}^{t} \frac{\partial}{\partial \mathbf{n}} g(r, \tau) \varphi(\mathbf{y}, \tau) d \tau d \Gamma \\
& =\int_{\Gamma} \int_{0}^{t} g(r, \tau) \psi(\mathbf{y}, \tau) d \tau d \Gamma
\end{aligned}
$$

where $c(\mathbf{x})$ is the solid angle at point $\mathbf{x}, \varphi(\mathbf{x}, t)$ is the velocity potential at point $\mathbf{x}$ at time $t, \Gamma$ denotes the acoustic domain boundary, $\mathbf{n}$ is the inward unit normal vector from fluid domain to $\Gamma, \psi(\mathbf{y}, \tau)=\frac{\partial}{\partial \mathbf{n}} \varphi(\mathbf{y}, \tau)$ represents flux, and $g(r, \tau)$ is the transient fundamental solution.

To solve the integral equation numerically, proper discretizations of the boundary $\Gamma$ and computation time $t$ are performed, and the unknowns on the boundary are interpolated using spatial and temporal basis functions. With the integral equation being discretized, the integrals

Corresponding author: xzhengzhang@hfut.edu.cn 
can be carried out numerically using standard Gaussian quadrature method. Finally, by assembling coefficients into corresponding global matrices, the following linear algebraic equations can be obtained

$$
\mathbf{C} \boldsymbol{\Phi}^{(n)}+\sum_{m=\mathbf{0}}^{n} \mathbf{H}^{(m)} \boldsymbol{\Phi}^{(n-m)}=\sum_{m=\mathbf{0}}^{n} \mathbf{G}^{(m)} \boldsymbol{\Psi}^{(n-m)}
$$

where $n$ represents the $\mathrm{n}$-th time step at $t_{n}=t_{0}+n \Delta t$, with $t_{\mathbf{0}}$ the initial time and $\Delta t$ the time step size. $\mathbf{C}$ is a diagonal matrix whose elements are the solid angles, the vectors $\boldsymbol{\Phi}^{(m)}$ and $\boldsymbol{\Psi}^{(m)}$ contain all the velocity potentials and fluxes at time $t_{m}$, respectively, and the matrices $\mathbf{H}^{(m)}$ and $\mathbf{G}^{(m)}$ consist of corresponding coefficients.

The ITBEM formulations can be derived based on Eq. (2), and the detailed derivation can be found in the work of Zhang et al. [14]. For concision, the ITBEM formulations are directly given here

$$
\begin{aligned}
& \left(\frac{1}{2}+\mathbf{H}_{s}^{(0)}\right) \boldsymbol{\Phi}_{s}^{(n)}-\mathbf{G}_{s}^{(0)} \boldsymbol{\Psi}_{s}^{(n)} \\
& =\sum_{m=1}^{n_{s x}}\left(\mathbf{G}_{s}^{(m)} \boldsymbol{\Psi}_{s}^{(n-m)}-\mathbf{H}_{s}^{(m)} \boldsymbol{\Phi}_{s}^{(n-m)}\right) \\
& \mathbf{H}_{f}^{\left(n_{f m}\right)} \boldsymbol{\Phi}_{s}^{(n)}-\mathbf{G}_{f}^{\left(n_{f m}\right)} \boldsymbol{\Psi}_{s}^{(n)} \\
& =\sum_{m=n_{f m}+1}^{n_{f x}}\left(\mathbf{G}_{f}^{(m)} \boldsymbol{\Psi}_{s}^{(\bar{n})}-\mathbf{H}_{f}^{(m)} \boldsymbol{\Phi}_{s}^{(\bar{n})}\right)-\boldsymbol{\Phi}_{f}^{\left(n+n_{f n}\right)}
\end{aligned}
$$

where $n_{s x}$ is the maximum non-zero surface matrices index, $n_{f m}$ and $n_{f x}$ are the minimum and maximum non-zero field matrices indexes, and $\bar{n}=n-m+n_{f m}$. The equations above can also be reformulated to a more concise form as

$$
\mathbf{A}^{(0)} \mathbf{X}^{(n)}=-\sum_{m=1}^{n_{s}} \mathbf{A}^{(m)} \mathbf{X}^{(n-m)}-\boldsymbol{\Phi}_{f}^{\left(n+n_{f m}\right)}
$$

where $n_{s}=\max \left(n_{s x}, n_{f x}-n_{f m}+1\right)$, and

$$
\begin{gathered}
\mathbf{X}^{(n)}=\left[\begin{array}{c}
\boldsymbol{\Phi}_{s}^{(n)} \\
\boldsymbol{\Psi}_{s}^{(n)}
\end{array}\right] \\
\mathbf{A}^{(n)}= \begin{cases}{\left[\begin{array}{cc}
\left(\frac{1}{2}+\mathbf{H}_{s}^{(0)}\right) & -\mathbf{G}_{s}^{(0)} \\
\mathbf{H}_{f}^{\left(n_{f i n}\right)} & -\mathbf{G}_{f}^{\left(n_{f m}\right)}
\end{array}\right], n=0} \\
{\left[\begin{array}{cc}
\mathbf{H}_{s}^{(n)} & -\mathbf{G}_{s}^{(n)} \\
\mathbf{H}_{f}^{\left(n+n_{f i n}\right)} & -\mathbf{G}_{f}^{\left(n+n_{f m}\right)}
\end{array}\right], n>0}\end{cases}
\end{gathered}
$$

By solving Eq. (5) in a MOT way with the measured potentials $\boldsymbol{\Phi}_{f}^{(n)}$, the surface potentials $\boldsymbol{\Phi}_{s}^{(n)}$ and fluxes $\boldsymbol{\Psi}_{s}^{(n)}$ can be reconstructed. With the surface potentials and fluxes known, the whole sound field can then be reconstructed. It should be noted that when solving Eq.
(5), the truncated singular value decomposition method should be used for stable and accurate results.

\section{Experiment of a tire}

To validate the proposed method, an experiment of a slick tire was conducted in a semi-anechoic chamber, as shown in Fig.1. The tire was supported away from the ground to get rid of the influence of the reflection. A steel sphere which was hung up as a simple pendulum was used to impact the tire for generating transient sound field. The sphere was controlled by a fixed electromagnet so that it was released at the same position at each measurement. An array of 7 microphones was used to scan the transient sound field, as shown in Fig.1(a). The measurement was triggered by an acceleration sensor which was stuck near the impact point on the surface of the tire so that all the measurement data was time-synchronized. To evaluate the reconstruction results, the acceleration on the surface of the tire was also measured, as shown in Fig.1(b).

The schematic setup of the experiment is shown in Fig.2. A Cartesian coordinate system is established with the origin $O$ at the geometry center of the tire. There are 273 measurement points evenly distributed on the measurement surface $\Gamma_{H}$ whose radius is $r_{m}=0.65 \mathrm{~m}$. The diameter $d_{c}$ and width $h_{c}$ of the tire can be calculated according to the type of the tire, which is 205/55R16. In Fig.2 (b), the positions of the measured acceleration, the impact point and the trigger are indicated by the points, the circle and the triangle, respectively.

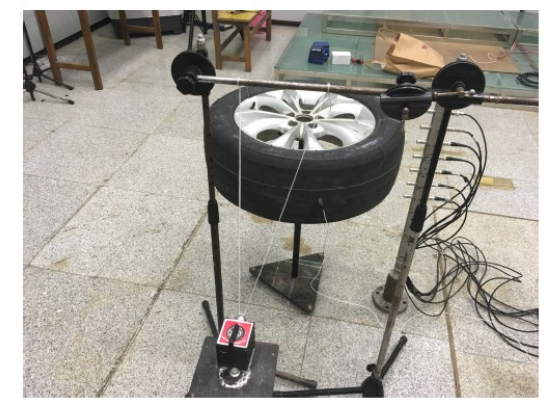

(a)

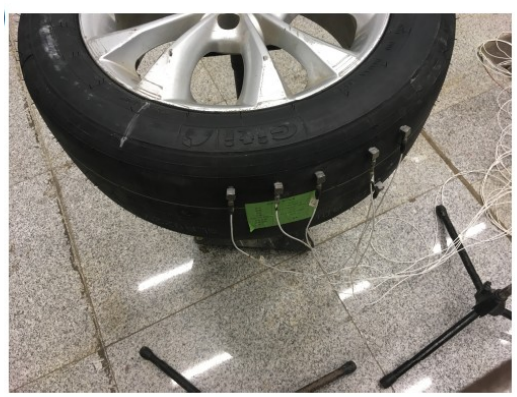

(b)

Fig. 1. Experiment scene: (a) pressure measurement, (b) accele ration measurement.

The spatial distributions of the measured pressure at variant instants are visualized in Fig.3. The first image in Fig. 3 shows the sound pressure at the very beginning of 
the impact. The impact position indicated by the pressure is consistent with that in Fig.2 (b). (a)

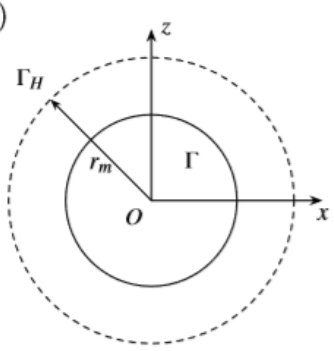

(b)

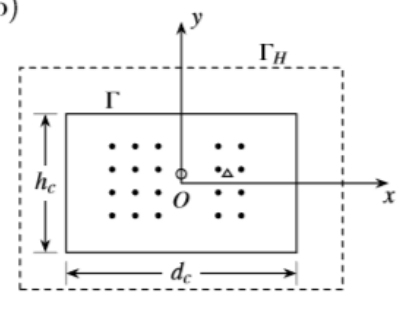

Fig. 2. Experiment setup: (a) top view, (b) front view.
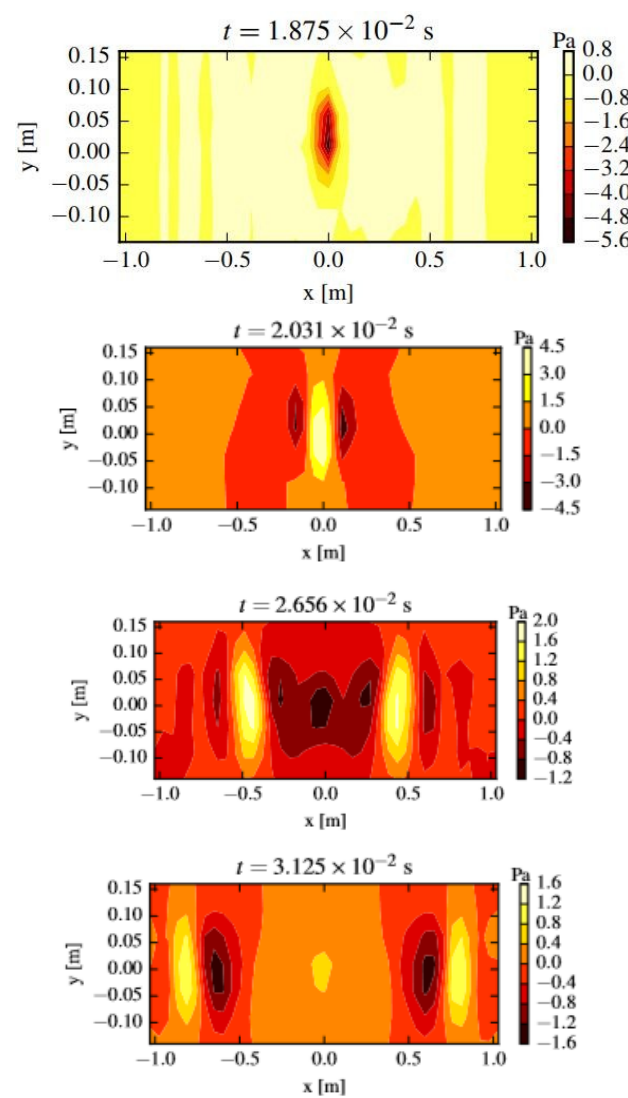

Fig. 3. Spatial distributions of measured pressure at 4 instants.

The pressure and acceleration on the surface of the tire are then reconstructed using ITBEM from the measured pressure. The numerical model of the tire is simplified to a cylinder with the same radius and width. A mesh of 1152 quadrilateral elements is adopted in the calculation. The time step size is chosen as $\Delta t=7.8 \times 10^{-5} \mathrm{~s}$. The spatial distributions of the reconstructed pressure and acceleration are given in Fig.4 and Fig.5, respectively. The reconstructed acceleration presents the vibration status of the tire surface after the impact. From the first image in Fig.5 the impact position can be identified directly. It can also be observed that the energy of the acceleration dissipates more quickly than that of the pressure, which exactly matches the common sense.
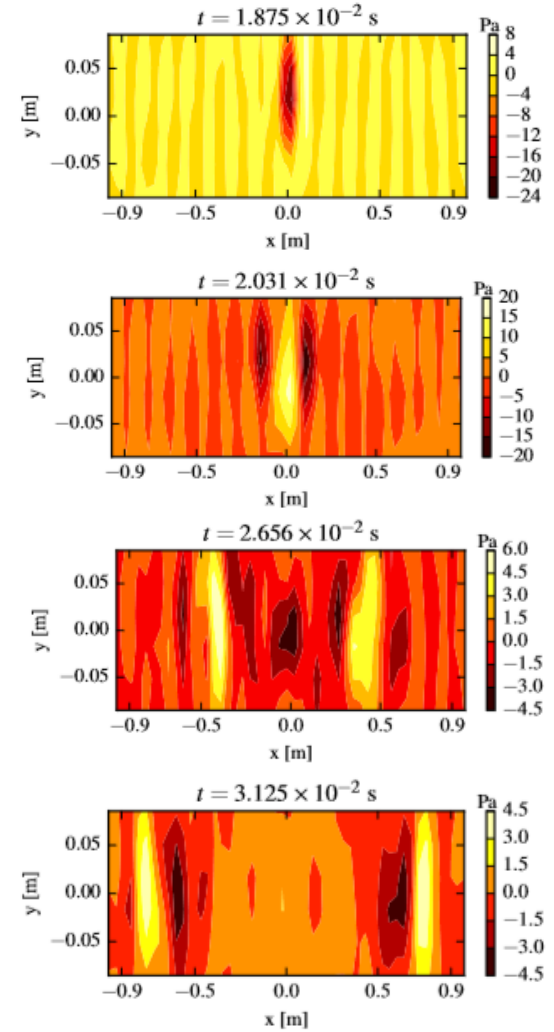

Fig. 4. Spatial distributions of reconstructed pressure at 4 instants.
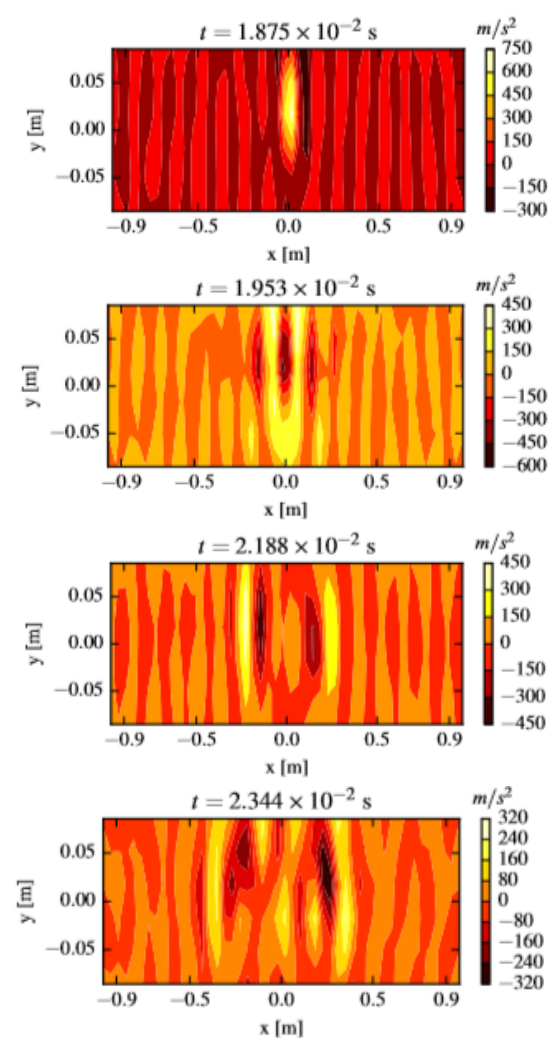

Fig. 5. Spatial distributions of reconstructed acceleration at 4 instants. 


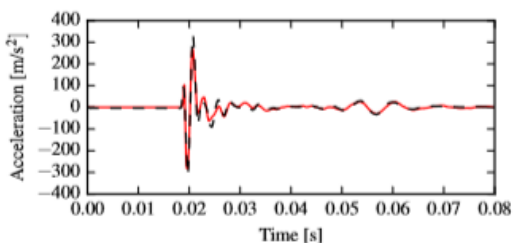

(a)

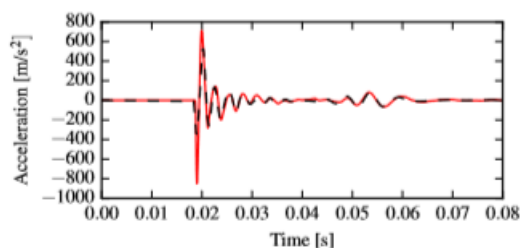

(b)

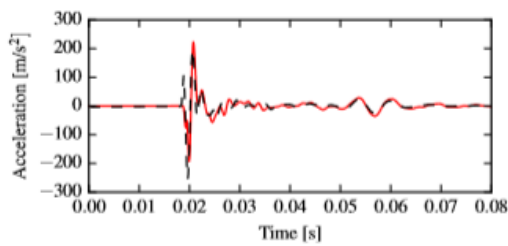

(c)

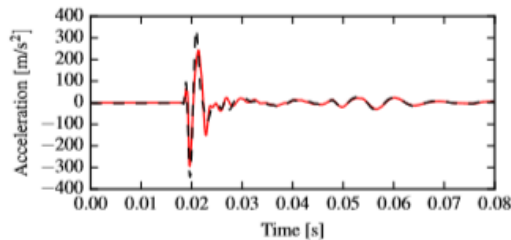

(d)

Fig. 6. Comparisons of reconstructed (dash line) and measured (solid line) acceleration.

To evaluate the reconstructed results more precisely, the reconstructed acceleration at 4 points are compared with the corresponding measured values, as shown in Fig.6. It can be seen that the waveforms of the reconstructed acceleration agree well with the measured values, although the magnitudes of the reconstructed results are overestimated or underestimated at some temporal points. The over- or under- estimation may come from the numerical errors which are caused by the difference between the numerical model and the real tire. The reconstructed results demonstrate that the ITBEM can reconstruct transient sound field effectively.

\section{Conclusions}

In this paper, the ITBEM is validated through an experiment of a tire. The tire is impacted by a sphere to generate a transient sound field. By visualizing the measured pressure, the wave propagation phenomenon can be seen clearly. The pressure and acceleration on the surface of the tire are reconstructed through ITBEM. Moreover, the reconstructed acceleration is compared to the measured values. The agreement between them demonstrates that the ITBEM is effective in reconstructing transient sound field.

This work was supported by the National Natural Science Foundation of China (Grant No.11674082 and No.51875147) and the Fundamental Research Funds for the Central Universities (Grant No.JZ2017HGTB0207).

\section{References}

1. E.G. Williams, J.D. Maynerd, Phys. Rev. Lett. 45, 554-557 (1980)

2. E.G. Williams, J.D. Maynerd, E. Skudrzky, J. Acoust. Soc. Am. 68, 340-344 (1980)

3. E.G. Williams, Fourier acoustics: sound radiation and nearfield acoustical holography (Academic Press, London, 1999)

4. B.K. Gardner, R.J. Bernhard, ASME J. Vib. Acoust. 110, 84-90 (1988)

5. W.A. Veronesi, J.D. Maynerd, J. Acoust. Soc. Am. 85, 588-598 (1989)

6. M.B. Friedman, R.P. Shaw, ASME J. App. Mech. 29, 40-46 (1962)

7. K.M. Mitzner, J. Acoust. Soc. Am. 42, 391-397 (1967)

8. Y. Ding, A. Forestier, T. Ha-Duong, J. Acoust. Soc. Am. 86, 1566-1572 (1989)

9. M.J. Bluck, S.P. Walker, Int. J. Numer. Meth. Eng. 39, 1419-1431 (1996)

10. D.J. Chappell, P.J. Harris, D. Henwood, R. Chakrabarti, J. Acoust. Soc. Am. 120, 74-80 (2006)

11. B. P. Rynne, Electromagnetics 6, 129-144 (1986)

12. A.A. Ergin, B. Shanker, E. Michielssen, J. Acoust. Soc. Am. 105, 2396-2404 (1999)

13. H.W. Jang, J.G. Ih, J. Acoust. Soc. Am. 131, $2742-$ 2752 (2012)

14. Y. Zhang, X.Z. Zhang, C.X. Bi, Y.B. Zhang, ASME J. Vib. Acoust. 139, 1-8 (2017)

15. W.J. Mansur, Ph.D. thesis, Southampton University, Southampton, UK (1983) 